\title{
Cu-catalyzed Tandem C-N Bond-Formation for the Synthesis of Pyrroles and Heteroarylpyrroles
}

Ruben Martin, Catharine H. Larsen, Ana Cuenca and Stephen L. Buchwald*

Department of Chemistry, Room 18-490

Massachusetts Institute of Technology

Cambridge, MA 02139 (USA)

FAX: (+1) 617-253-3297

E-mail: sbuchwal@ mit.edu

\section{Supporting Information}

\section{General Considerations}

Reagents. All reactions were set up on the benchtop and carried out under an argon atmosphere in oven-dried Schlenk tubes. Cul was purchased from Strem Chemicals. Powdered $\mathrm{Cs}_{2} \mathrm{CO}_{3}$ was a gift from Chemetall. Anhydrous $\mathrm{K}_{2} \mathrm{CO}_{3}$ was purchased from Mallinckrodt Chemicals. The bulk of both bases were stored under nitrogen in a Vacuum Atmospheres glovebox. Small quantities $(\sim 5 \mathrm{~g})$ were removed from the glovebox in glass vials, stored in the air in a desiccator (anhydrous calcium sulfate), and weighed out on the benchtop. $N, N^{\prime}$-dimethylethylenediamine was obtained from Aldrich. Commercially available materials were used without further purification unless otherwise noted. Toluene and THF were purchased from J. T. Baker in CYCLE-TAINER solvent delivery kegs, which were vigorously purged with argon for $2 \mathrm{~h}$, and further purified by passing the solvent through two packed columns of neutral alumina and copper (II) oxide under argon pressure. All other reagents were purchased from commercial sources and used as received. Flash chromatography was performed with EM Science silica gel 60 (230-400 mesh).

Analytical Methods. All new compounds were characterized by ${ }^{1} \mathrm{H} N M R,{ }^{13} \mathrm{C} N M R, \mathrm{IR}$ spectroscopy and in most cases, elemental analysis. ${ }^{1} \mathrm{H}$ NMR and ${ }^{13} \mathrm{C}$ NMR spectra and melting points (where applicable) are included for all known compounds and for all new compounds not characterized by elemental analysis. ${ }^{1} \mathrm{H}$ and ${ }^{13} \mathrm{C}$ NMR spectra were recorded on a Bruker $400 \mathrm{MHz}$. Infrared spectra were recorded on a Perkin-Elmer Model $2000 \mathrm{FT}-\mathrm{IR}$ using $\mathrm{NaCl}$ plates (thin film). Elemental analyses were performed by Atlantic Microlab Inc., Norcross, GA. All ${ }^{1} \mathrm{H}$ NMR spectra are reported in parts per million (ppm) downfield of TMS and were measured relative to the signals for $\mathrm{CHCl}_{3}(7.27 \mathrm{ppm})$. All ${ }^{13} \mathrm{C}$ 
NMR spectra were reported in ppm relative to residual $\mathrm{CHCl}_{3}(77 \mathrm{ppm})$ and were obtained with ${ }^{1} \mathrm{H}$ decoupling. Melting points $(\mathrm{Mp})$ were obtained on a Mel-Temp capillary melting point apparatus. Gas chromatographic analyses were performed on Hewlett-Packard 6890 gas chromatography instrument with a FID detector using $25 \mathrm{~m} \times 0.20 \mathrm{~mm}$ capillary column with cross-linked methyl siloxane as the stationary phase. The yields reported in tables 1 and Scheme 5 refer to isolated yields and represent an average of at least two independent runs. The pure compounds are estimated to be $\geq 95 \%$ pure as determined by ${ }^{1} \mathrm{H}$ NMR and GC analysis and/or combustion analysis. The procedures described in this section are representative.

\section{Synthesis of the starting materials}




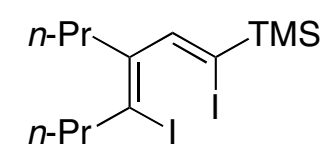

[(1Z,3Z)-1,4-Diiodo-3-propyl-1,3-heptadienyl](trimethyl)silane (1a). Following the literature procedure, ${ }^{1}$ using 3-octyne $(1.62 \mathrm{~mL}, 11.0 \mathrm{mmol})$ and trimethylsilyl acetylene (1.42 mL, $10.0 \mathrm{mmol})$. Column chromatography: silica gel, hexanes. Colorless oil; yield: $3.74 \mathrm{~g}(81 \%)$. The product was purified by column chromatography (silica gel, hexanes) to give $3.74 \mathrm{~g}$ of the title compound ( $81 \%$ yield) as a colorless oil. ${ }^{1} \mathrm{H}$ NMR $\left(400 \mathrm{MHz}, \mathrm{CDCl}_{3}\right)$ ס: 6.84 (s, $1 \mathrm{H}), 2.58$ (t, J=7.2 Hz, 2H), 2.40 (t, J=7.6 Hz, 2H), $1.64(\mathrm{~m}, 2 \mathrm{H}), 1.42(\mathrm{~m}, 2 \mathrm{H})$, 1.01 (t, J=7.2 Hz, 3H), 0.93 (t, J=7.2 Hz, 3H), 0.25 (s, 9H). ${ }^{13} \mathrm{C} \mathrm{NMR}\left(100 \mathrm{MHz}, \mathrm{CDCl}_{3}\right) \delta$ : 151.7, 145.7, 114.0, 106.1, 41.9, 34.2, 22.9, 21.8, 14.2, 13.1, -1.5. IR (neat, $\mathrm{cm}^{-1}$ ): 2959, 2930, 1581, 1458, 1378, 1247, 1188, 1099, 897, 841, 753.

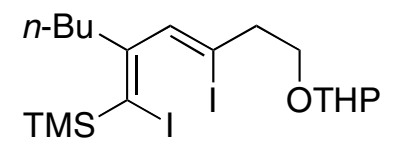

[(1Z,3Z)-2-Butyl-1,4-diiodo-6-(tetrahydro-2H-pyran-2-yloxy)-1,3-hexadienyl](trimethyl) silane (1c). Following the literature procedure, ${ }^{1}$ using 2-but-3-ynyloxy-tetrahydropyran (1.56 mL, $10.0 \mathrm{mmol}$ ) and 1-trimethylsilyl-1-hexyne (2.22 mL, $11.0 \mathrm{mmol})$. Column chromatography: silica gel, 10:1 hexanes/ethyl acetate. Colorless oil; yield: $2.93 \mathrm{~g} \mathrm{(51 \% ).}$ ${ }^{1} \mathrm{H} \mathrm{NMR}\left(400 \mathrm{MHz}, \mathrm{CDCl}_{3}\right)$ ס: $6.31(\mathrm{~s}, 1 \mathrm{H}), 4,68(\mathrm{~m}, 2 \mathrm{H}), 3.89(\mathrm{~m}, 2 \mathrm{H}), 3.63(\mathrm{~m}, 2 \mathrm{H}), 3.42$ (m, 2H), $2.69(\mathrm{~m}, 4 \mathrm{H}), 2.32(\mathrm{t}, \mathrm{J}=8 \mathrm{~Hz}, 2 \mathrm{H}), 2.22(\mathrm{t}, \mathrm{J}=7.7 \mathrm{~Hz}, 2 \mathrm{H}), 1.55-1.31(\mathrm{~m}, 22 \mathrm{H})$, 0.91 (t, J=7.6 Hz, 3H), 0.31 (s, 9H). ${ }^{13} \mathrm{C}$ NMR $\left(100 \mathrm{MHz}, \mathrm{CDCl}_{3}\right) \delta: 144.4,137.3,114.3$, 98.7, 93.8, 92.3, 87.2, 82.4, 65.5, 62.0, 38.8, 37.3, 31.5, 30.5, 25.4, 22.3, 21.1, 19.3, 14.0, 1.9. IR (neat, $\mathrm{cm}^{-1}$ ): 2951, 2932, 2877, 1630, 1557, 1457, 1373, 1234, 1171, 1056, 1041, 988, 855, 815, 779, 726, 702. Anal. Calcd for $\mathrm{C}_{18} \mathrm{H}_{32} \mathrm{l}_{2} \mathrm{O}_{2} \mathrm{Si}: \mathrm{C}, 38.45 ; \mathrm{H}, 5.74$. Found: $\mathrm{C}$, 38.19; H, 5.88 .



[(1Z,3Z)-2-Butyl-8-chloro-1,4-diiodo-1,3-octadienyl](trimethyl)silane (1d). Following the literature procedure, ${ }^{1}$ using 6-chloro-1-hexyne $(1.23 \mathrm{~mL}, 10.0 \mathrm{mmol})$ and 1 trimethylsilyl-1-hexyne (2.22 mL, $11.0 \mathrm{mmol})$. Column chromatography: silica gel, hexanes. Colorless oil; yield: $2.78 \mathrm{~g}(53 \%)$. ${ }^{1} \mathrm{H}$ NMR $\left(400 \mathrm{MHz}, \mathrm{CDCl}_{3}\right)$ ס: $6.27(\mathrm{~s}, 1 \mathrm{H}), 3.59$ (t, J=7.2 $\mathrm{Hz}, 2 \mathrm{H}), 2.61$ (t, J=6.8 Hz, 2H), $2.47(\mathrm{t}, \mathrm{J}=7.2 \mathrm{~Hz}, 2 \mathrm{H}), 1.87(\mathrm{~m}, 2 \mathrm{H}), 1.78(\mathrm{~m}, 2 \mathrm{H}), 1.41(\mathrm{~m}$, $2 \mathrm{H}), 1.30(\mathrm{~m}, 2 \mathrm{H}), 0.92(\mathrm{t}, \mathrm{J}=7.2 \mathrm{~Hz}, 3 \mathrm{H}), 0.34(\mathrm{~s}, 9 \mathrm{H}) .{ }^{13} \mathrm{C} \mathrm{NMR}\left(100 \mathrm{MHz}, \mathrm{CDCl}_{3}\right) \delta$ :

\footnotetext{
${ }^{1}$ Buchwald, S. L.; Nielsen, R. B. J. Am. Chem. Soc. 1989, 111, 2870.
} 
$158.8,142.4,109.8,108.7,44.7,43.6,36.5,31.2,26.6,22.8,13.9,1.8 . \mathrm{IR}$ (neat, $\mathrm{cm}^{-1}$ ): 2955, 2928, 2859, 1634, 1575, 1456, 1377, 1301, 1249, 1091, 841, 758, 689, 624. Anal. Calcd for $\mathrm{C}_{15} \mathrm{H}_{27} \mathrm{Cll}_{2} \mathrm{Si}: \mathrm{C}, 34.33 ; \mathrm{H}, 5.19$. Found: C, 34.27; $\mathrm{H}, 5.29$.



\section{Di(tert-butyl)(4E,5E)-4,5-bis[iodo(trimethylsilyl)methylene]tetrahydro-1,2-pyradizine}

dicarboxylate $(1 \mathrm{~m})$. Following the literature procedure, ${ }^{2}$ using di(tert-butyl)1,2-bis[3(trimethylsilyl)-2-propynyl]-1,2-hydrazine dicarboxylate ${ }^{3}(1.00 \mathrm{~g}, 2.21 \mathrm{mmol})$. Column chromatography: silica gel, 10:1 hexanes/ethyl acetate. White solid; yield: $0.64 \mathrm{~g}(41 \%)$. Mp 98-99 ${ }^{\circ} \mathrm{C} .{ }^{1} \mathrm{H}$ NMR $\left(400 \mathrm{MHz}, \mathrm{CDCl}_{3}\right)$ ס: 4.62-4.35 (m, 4H), 1.49-1.45 (m, 18H), 0.40$0.33(\mathrm{~m}, 18 \mathrm{H})$ (conformers). ${ }^{13} \mathrm{C}$ NMR $\left(100 \mathrm{MHz}, \mathrm{CDCl}_{3}\right) \delta: 155.3,154.2,109.7,109.3$, 81.8, 81.5, 53.0, 51.8, 28.4, 28.3, 1.2, 1.1. IR (neat, $\mathrm{cm}^{-1}$ ): 2976, 1710, 1577, 1455, 1391, $1367,1251,1169,1127,1024,857,843$. Anal. Calcd for $\mathrm{C}_{22} \mathrm{H}_{40} \mathrm{I}_{2} \mathrm{~N}_{2} \mathrm{O}_{4} \mathrm{Si}_{2}: \mathrm{C}, 37.40 ; \mathrm{H}$, 5.71. Found: C, 37.49; $\mathrm{H}, 5.58$.<smiles>C=C(C)C(/C=C(\I)c1ccsc1)=C(/C)I</smiles>

\section{[(1E,3Z)-1,4-diiodo-2-isopropenyl-4-(3-thienyl)-1,3-butadienyl](trimethyl)silane}

(1f).

Following the literature procedure, ${ }^{4}$ using trimethyl-(3-methyl-but-3-en-1-ynyl)-silane (0.94 $\mathrm{g}, 6.81 \mathrm{mmol}$ ) and 3-ethynylthiophene $(0.57 \mathrm{~mL}, 5.79 \mathrm{mmol})$. Column chromatography: silica gel, hexanes. Yellow oil; yield: $1.65 \mathrm{~g} \mathrm{(57 \% ).}{ }^{1} \mathrm{H} \mathrm{NMR}\left(400 \mathrm{MHz}, \mathrm{CDCl}_{3}\right)$ ס: 7.53 (t, $J=1.6 \mathrm{~Hz}, 1 \mathrm{H}$ ), 7.39 (d, J=2.8 Hz, 1H), $7.36(\mathrm{~d}, J=0.9 \mathrm{~Hz}, 1 \mathrm{H}), 6.72(\mathrm{~s}, 1 \mathrm{H}), 5.18(\mathrm{~s}, 1 \mathrm{H})$, $5.06(\mathrm{~s}, 1 \mathrm{H}), 1.91$ (s, 3H), $0.33(\mathrm{~s}, 9 \mathrm{H}) .{ }^{13} \mathrm{C} \mathrm{NMR}\left(100 \mathrm{MHz}, \mathrm{CDCl}_{3}\right)$ ס: 159.9, 144.3, 143.4, 140.0, 126.0, 125.9, 125.6, 119.2, 115.3, 99.8, 22.4, 1.7. IR (neat, $\mathrm{cm}^{-1}$ ): 2965, 2912, 2856, 1559, 1404, 1369, 1247, 1168, 900, 844, 779. Anal. Calcd for $\mathrm{C}_{14} \mathrm{H}_{18} \mathrm{l}_{2} \mathrm{SSi}$ : C, 33.61; $\mathrm{H}$, 3.63. Found: C, 33.49; H, 3.78 .

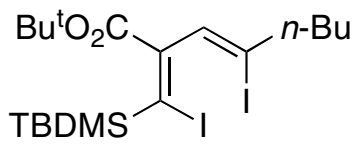

\footnotetext{
${ }^{2}$ Yamaguchi, S.; Jin, R-Z.; Tamao, K.; Sato, F. J. Org. Chem. 1998, 63, 10060.

${ }^{3}$ Rasmussen, L. K. J. Org. Chem. 2006, 71, 3627.

${ }^{4}$ Hamada, T.; Suzuki, D.; Urabe, H.; Sato, F. J. Am. Chem. Soc. 1999, 121, 7342.
} 
tert-Butyl (2E,3Z)-2-[[tert-butyl(dimethyl)silyl](iodo)methylene]-4-iodo-3-octenoate (1e). Following the literature procedure, ${ }^{4}$ using (tert-butyl-dimethylsilyl)-propynoic acid tertbutyl ester $(0.80 \mathrm{~g}, 3.33 \mathrm{mmol})$ and 1-hexyne $(0.31 \mathrm{~mL}, 2.67 \mathrm{mmol})$. Column chromatography: silica gel, 30:1 hexanes/ethyl acetate. Colorless oil; yield: $0.97 \mathrm{~g}(63 \%)$. ${ }^{1} \mathrm{H}$ NMR (400 MHz, $\mathrm{CDCl}_{3}$ ) $\delta: 6.32(\mathrm{~s}, 1 \mathrm{H}), 2.60(\mathrm{t}, \mathrm{J}=7.1 \mathrm{~Hz}, 2 \mathrm{H}), 1.61-1.55(\mathrm{~m}, 2 \mathrm{H}), 1.48$ (s, 9H), 1.44-1.39 (m, 2H), $1.10(\mathrm{~s}, 9 \mathrm{H}), 0.94(\mathrm{t}, \mathrm{J}=7.2 \mathrm{~Hz}, 3 \mathrm{H}) .{ }^{13} \mathrm{C}$ NMR $\left(100 \mathrm{MHz}, \mathrm{CDCl}_{3}\right)$ ঠ: $162.9,153.2,139.6,124.4,112.0,81.9,44.3,31.3,28.3,26.0,21.3,18.7,13.8,-0.7$. IR (neat, $\mathrm{cm}^{-1}$ ): 2957, 2929, 2857, 1724, 1634, 1542, 1469, 1392, 1367, 1249, 1166, 1138, 843, 824, 808, 776. Anal. Calcd for $\mathrm{C}_{19} \mathrm{H}_{34} \mathrm{I}_{2} \mathrm{O}_{2} \mathrm{Si}$ : C, 39.59; $\mathrm{H}, 5.95$. Found: C, 39.70; $\mathrm{H}$, 5.88 .<smiles>CCCCC(=C(I)C(C)=[W])C(C)C</smiles>

[(1Z,3Z)-2-butyl-1,4-diiodo-3-propyl-1,3-heptadienyl](trimethyl)silane (1h). Following the literature procedure, ${ }^{4}$ using 3-octyne $(2 \mathrm{~mL}, 13.61 \mathrm{mmol})$ and 1-trimethylsilyl-1-hexyne (2.34 mL, $11.57 \mathrm{mmol})$. Column chromatography: silica gel, hexanes. Colorless oil; yield: $2.09 \mathrm{~g}(35 \%)$. ${ }^{1} \mathrm{H}$ NMR (400 MHz, $\left.\mathrm{CDCl}_{3}\right)$ ס: 2.73-2.13 (m, 6H), 1.78-1.33 (m, 8H), 1.09$0.92(\mathrm{~m}, 9 \mathrm{H}), 0.36(\mathrm{~s}, 6 \mathrm{H}) .{ }^{13} \mathrm{C}$ NMR $\left(100 \mathrm{MHz}, \mathrm{CDCl}_{3}\right) \delta: 163.4,150.0,109.6,106.8,42.5$, 36.6, 35.1, 31.0, 23.2, 22.7, 21.9, 14.8, 13.8, 13.4, 1.8. IR (neat, $\mathrm{cm}^{-1}$ ): 2959, 2871, 1456, 1377, 1248, 1120, 841, 758. Anal. Calcd for $\mathrm{C}_{17} \mathrm{H}_{32} \mathrm{l}_{2} \mathrm{Si}$ : C, 39.39; $\mathrm{H}, 6.22$. Found: $\mathrm{C}, 39.58$; $\mathrm{H}, 6.09$.

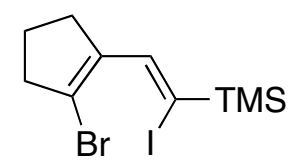

[(Z)-2-(2-Bromo-1-cyclopenten-1-yl)-1-iodoethenyl](trimethyl)silane (1j). DIBALH (4.89 $\mathrm{mL}, 4.89 \mathrm{mmol}, 1 \mathrm{M}$ in hexanes) was added dropwise to a solution of (2-Bromo-cyclopent1-enylethynyl)-trimethylsilane ${ }^{5}(0.78 \mathrm{~g}, 3.22 \mathrm{mmol})$ in pentanes $(10 \mathrm{~mL})$. The mixture was then stirred overnight at room temperature and $N$-iodosuccinimide $(1.11 \mathrm{~g}, 4.93 \mathrm{mmol})$ was added in portions over 5 minutes while the temperature was kept at $-20^{\circ} \mathrm{C}$. The slurry was stirred for an additional $6 \mathrm{~h}$ at $-20{ }^{\circ} \mathrm{C}$ and then water $(10 \mathrm{~mL})$ and hexanes $(10 \mathrm{~mL})$ were added consecutively. The combined organic layers were dried $\left(\mathrm{MgSO}_{4}\right)$ and reduced in vacuo. The crude product was purified by column chromatography on silica gel (hexanes) to give the title compound as a yellow oil (1.04 g, 87\%). ${ }^{1} \mathrm{H} \mathrm{NMR}\left(400 \mathrm{MHz}, \mathrm{CDCl}_{3}\right)$ ס: 7.37

\footnotetext{
${ }^{5}$ Kosinski, C.; Hirsch, A.; Heinemann, F. W.; Hampel, F. Eur. J. Org. Chem. 2001, 3879.
} 
(s, 1H), $3.03(\mathrm{t}, J=7.6 \mathrm{~Hz}, 2 \mathrm{H}), 2.65(\mathrm{t}, \mathrm{J}=7.6 \mathrm{~Hz}, 2 \mathrm{H}), 1.99(\mathrm{~m}, 2 \mathrm{H}), 0.26(\mathrm{~s}, 9 \mathrm{H}) .{ }^{13} \mathrm{C} \mathrm{NMR}$ $\left(100 \mathrm{MHz}, \mathrm{CDCl}_{3}\right) \delta: 139.3,138.7,127.4,110.8,40.2,33.2,22.3,-1.3$. IR (neat, $\mathrm{cm}^{-1}$ ): 2957, 2848, 1737, 1597, 1556, 1437, 1248, 1084, 912, 881, 839, 812, 753.

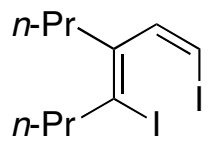

(1Z,3Z)-1,4-Diiodo-3-propyl-1,3-heptadiene (1b). TBAF (4.50 mL, $4.50 \mathrm{mmol}, 1 \mathrm{M} \mathrm{THF})$ was added dropwise to a solution of $1 \mathrm{a}(0.92 \mathrm{~g}, 1.98 \mathrm{mmol})$ in $\mathrm{THF}(10 \mathrm{~mL})$ at $0{ }^{\circ} \mathrm{C}$. The mixture was stirred for $30 \mathrm{~min}$, at which time water $(10 \mathrm{~mL})$ and hexanes $(10 \mathrm{~mL})$ were added. The combined organic layers were dried $\left(\mathrm{MgSO}_{4}\right)$ and evaporated. The crude product was purified by column chromatography on silica gel (hexanes) to give the title compound as a colorless oil $(0.54 \mathrm{~g}, 70 \%)$. ${ }^{1} \mathrm{H}$ NMR $\left(400 \mathrm{MHz}, \mathrm{CDCl}_{3}\right) \delta: 6.90$ (d, J=8.4 Hz, $1 \mathrm{H}), 6.41$ (d, J=8.4 Hz, 1H), 2.60 (t, J=7.2 Hz, 2H), 2.40 (t, J=7.6 Hz, 2H), 1.63 (m, 2H), $1.44(\mathrm{~m}, 2 \mathrm{H}), 1.00$ (t, J=7.2 Hz, 3H), 0.92 (t, J=7.2 Hz, 3H). ${ }^{13} \mathrm{C}$ NMR (100 MHz, CDCl $)_{3}$ ठ: 145.8, 143.2, 107.6, 83.5, 43.0, 34.1, 22.9, 21.8, 14.0, 13.0. IR (neat, $\mathrm{cm}^{-1}$ ): 2958, 2928, 2869, 1634, 1462, 1378, 1290, 1234, 1141, 1098, 909. 

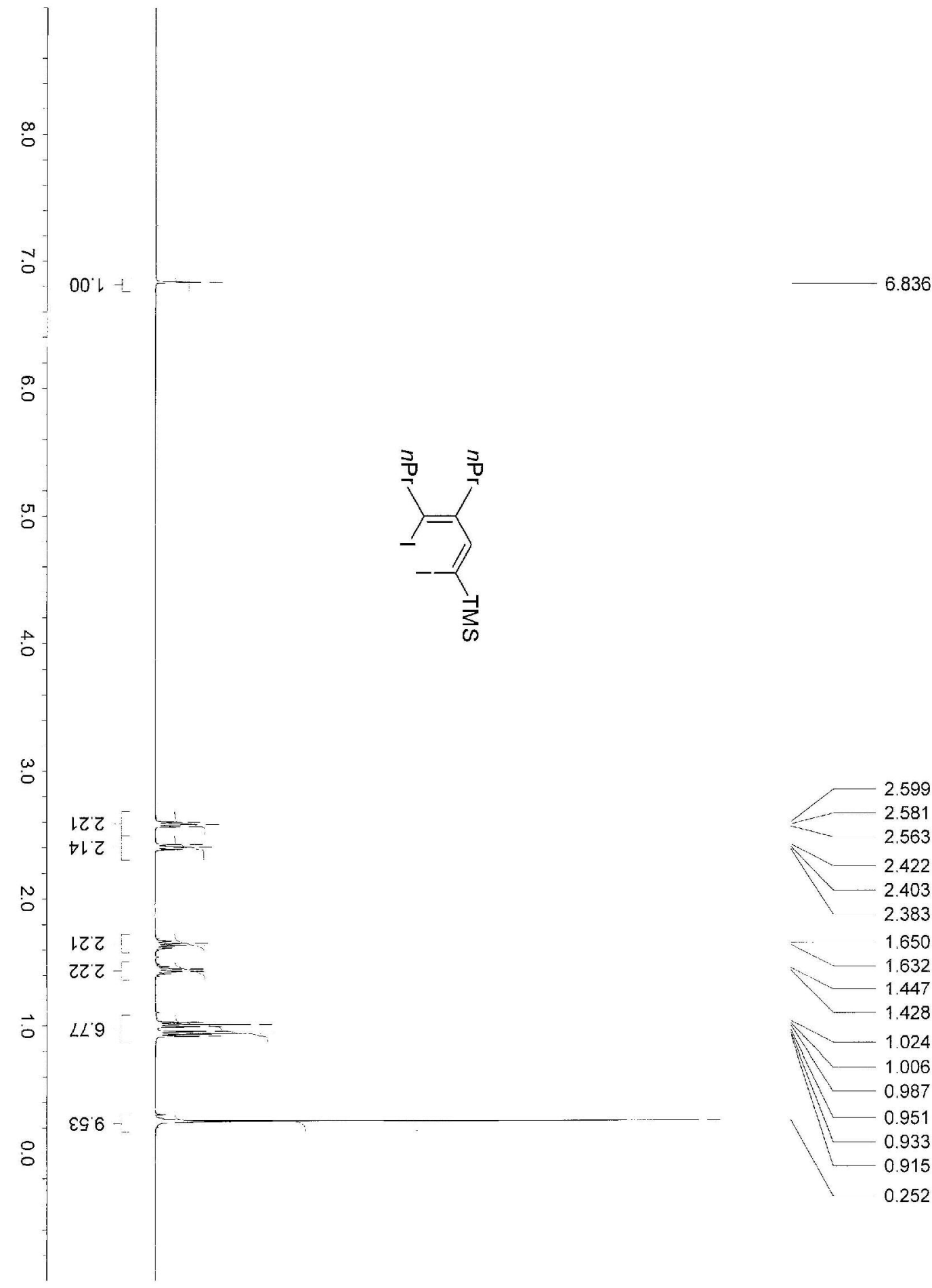


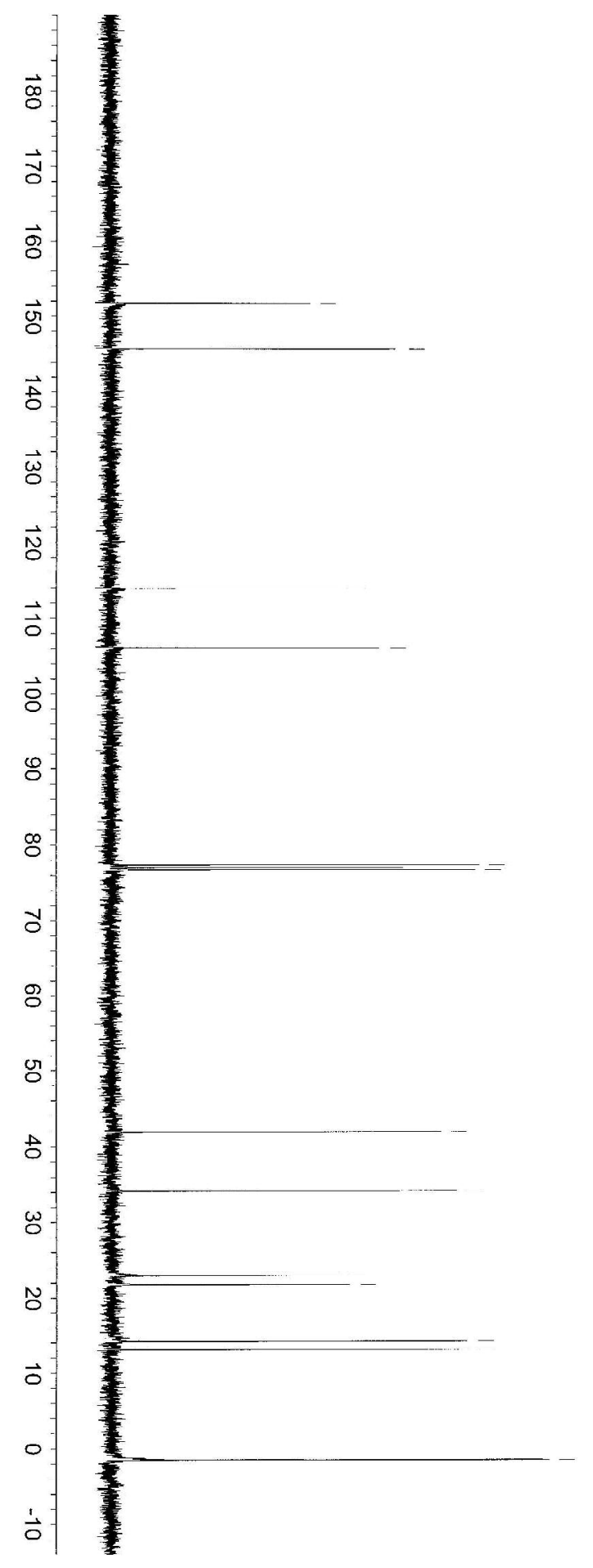

151.724

145.715

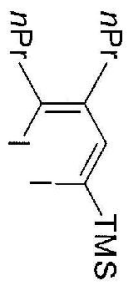

113.958

106.124

77.331

77.013

76.697

41.917

34.158

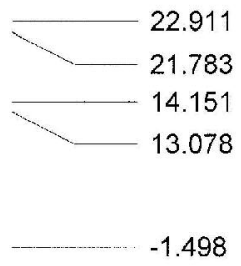





0.259 

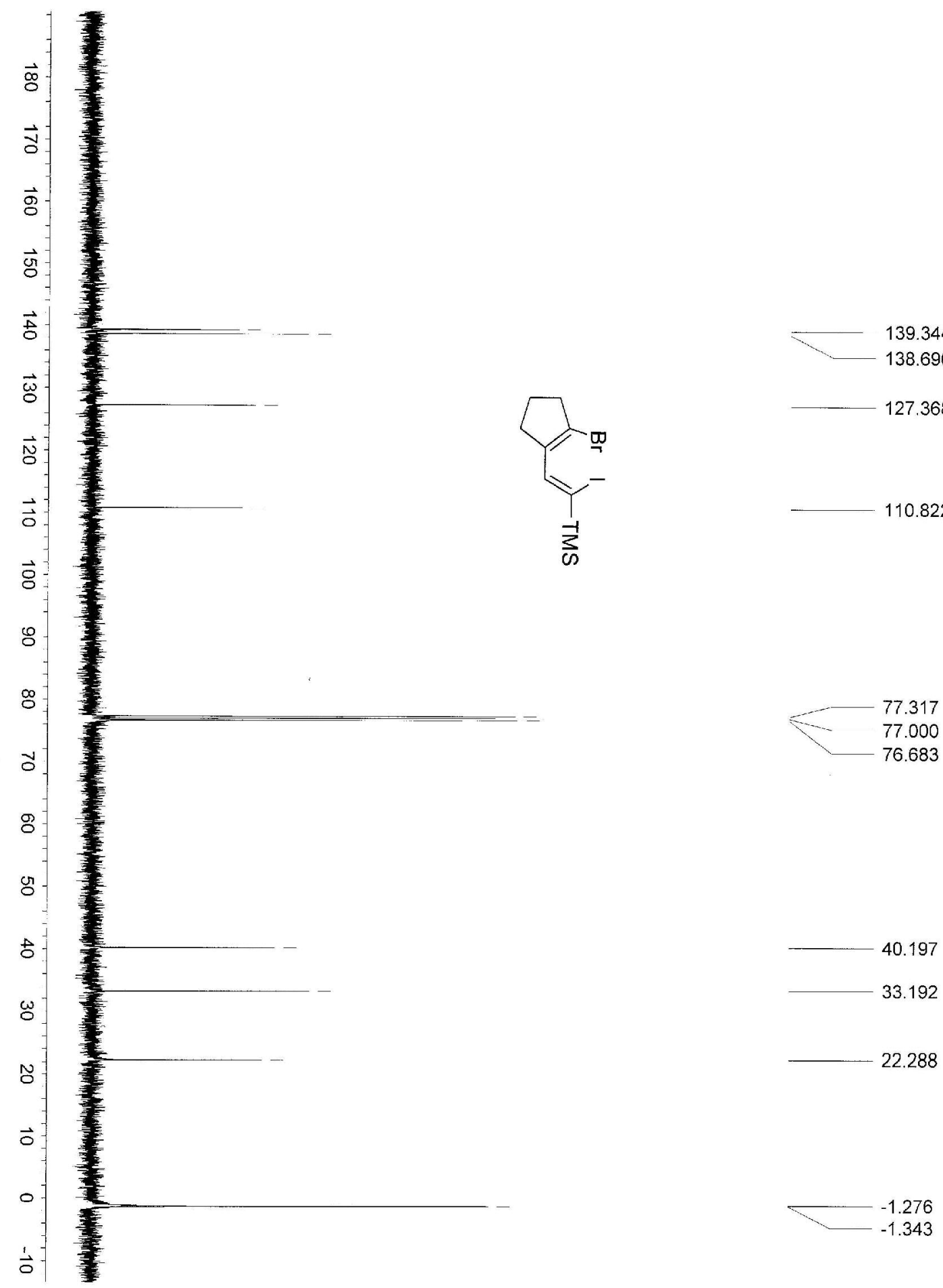

40.197

33.192

22.288

$-1.276$

$-1.343$ 



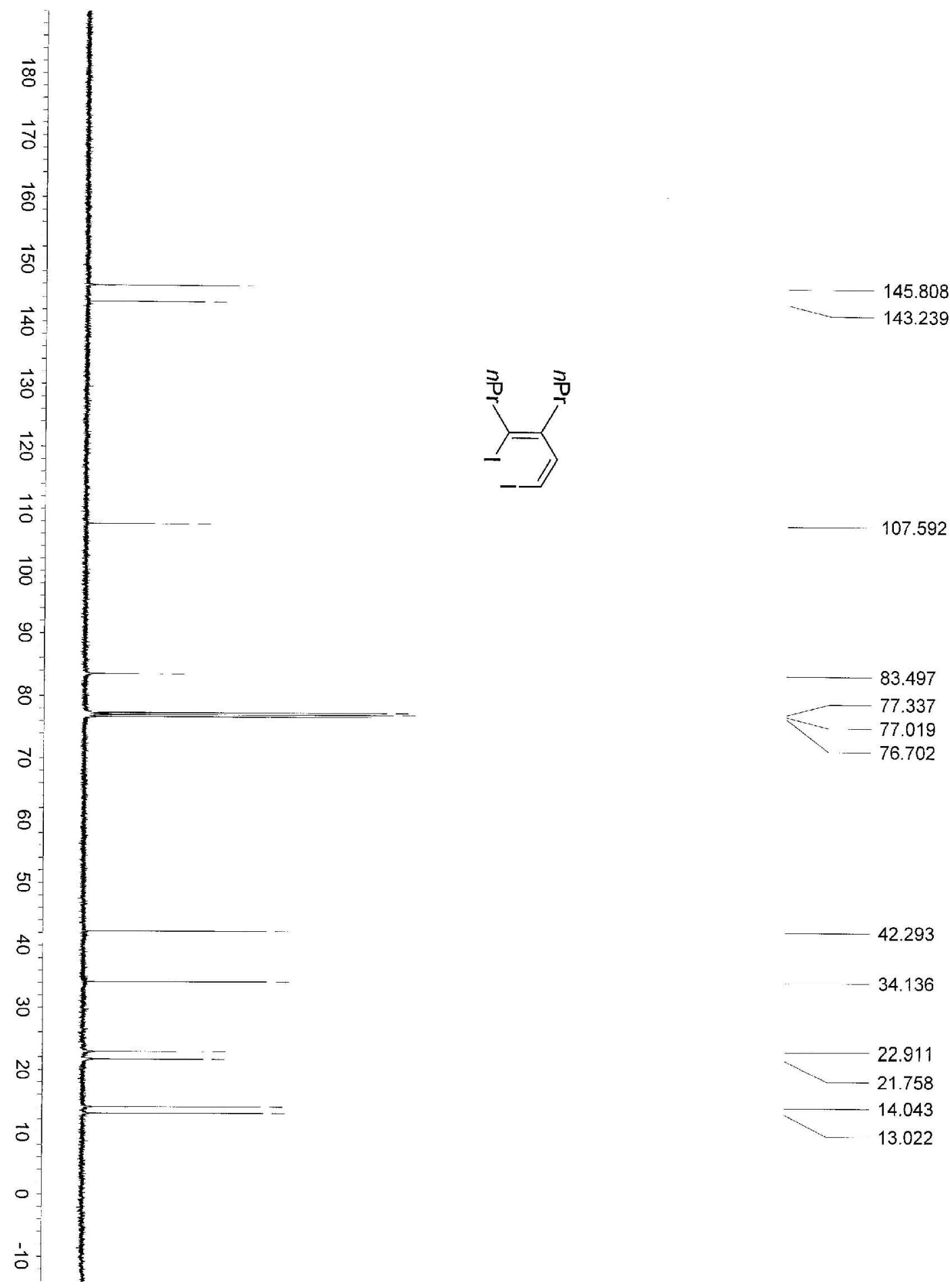

42.293

$-34.136$

22.911

21.758

14.043

13.022 


\section{Synthesis of Pyrroles}

General Procedure A for the synthesis of pyrroles through Cu-catalyzed sequential C-N bond-forming reactions (Table 1). An oven-dried Schlenk tube was charged with Cul (5 mol\%), 1,4-dihalo-1,3-diene (1 equiv., if a solid) tert-butyl carbamate (1.2 equiv.) and $\mathrm{Cs}_{2} \mathrm{CO}_{3}$ (3 equiv.). The Schlenk tube was capped with a teflon screwcap and then evacuated and backfilled with argon (this sequence was carried out two times). Under a positive pressure of argon, $N, N^{\prime}$-dimethylethylenediamine (20 mol\%) and 1,4-dihalo-1,3diene ( 1 equiv., if an oil) were added via syringe, followed by the addition of THF (0.5 M). The tube was sealed and stirred at $80^{\circ} \mathrm{C}$ in a pre-heated oil bath for the indicated period of time. The reaction mixture was cooled to room temperature, diluted with ethyl acetate, filtered through a plug of celite, concentrated to dryness and purified by column chromatography on silica gel $\left(2.5 \% \mathrm{NEt}_{3}\right)$, eluting with hexanes/ethyl acetate mixtures.

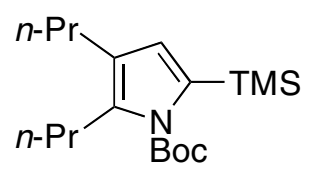

tert-Butyl 2,3-dipropyl-5-(trimethylsilyl)-1H-pyrrole-1-carboxylate (Table 1, 2a). Following general procedure A: $1 \mathrm{a}(0.46 \mathrm{~g}, 1.00 \mathrm{mmol})$, for $6 \mathrm{~h}$. Column chromatography: silica gel (2.5\% $\left.\mathrm{NEt}_{3}\right), 40: 1$ hexanes/ethyl acetate . Colorless oil; yield: $0.29 \mathrm{~g}(89 \%$ yield). ${ }^{1} \mathrm{H}$ NMR (400 MHz, $\mathrm{CDCl}_{3}$ ) $\delta: 6.34(\mathrm{~s}, 1 \mathrm{H}), 2.76(\mathrm{t}, \mathrm{J}=7.6 \mathrm{~Hz}, 2 \mathrm{H}), 2.36$ (t, J=7.6 Hz, 2H), $1.65(\mathrm{~s}, 9 \mathrm{H}), 1.57(\mathrm{~m}, 4 \mathrm{H}), 1.01(\mathrm{~m}, 6 \mathrm{H}), 0.30(\mathrm{~s}, 9 \mathrm{H}) .{ }^{13} \mathrm{C} \mathrm{NMR}\left(100 \mathrm{MHz}, \mathrm{CDCl}_{3}\right) \delta: 151.0$, 134.5, 134.7, 125.5, 123.1, 83.1, 28.8, 28.3, 28.0, 24.0, 23.9, 14.3, 14.1, 0.3. IR (neat, $\mathrm{cm}^{-}$ $\left.{ }^{1}\right): 2961,2872,1728,1457,1393,1357,1246,1151,1131,1007$, 842. Anal. Calcd for $\mathrm{C}_{18} \mathrm{H}_{33} \mathrm{NO}_{2} \mathrm{Si}: \mathrm{C}, 66.82 ; \mathrm{H}, 10.28$. Found: $\mathrm{C}, 66.93 ; \mathrm{H}, 10.11$.<smiles>CCCc1ccn(C(C)C)c1C(=O)O</smiles>

tert-Butyl 2,3-dipropyl-1H-pyrrole-1-carboxylate (Table 1, 2b). Following general procedure A: 1b $(0.20 \mathrm{~g}, 0.50 \mathrm{mmol})$, for $5 \mathrm{~h}$. Column chromatography: silica gel $(2.5 \%$ $\mathrm{NEt}_{3}$ ), 40:1 hexanes/ethyl acetate . Colorless oil; yield: $92 \mathrm{mg}$ (73\% yield). ${ }^{1} \mathrm{H}$ NMR (400 $\left.\mathrm{MHz}, \mathrm{CDCl}_{3}\right) \delta: 7.17(\mathrm{~d}, J=3.6 \mathrm{~Hz}, 1 \mathrm{H}), 6.02(\mathrm{~d}, J=3.6 \mathrm{~Hz}, 1 \mathrm{H}), 2.78(\mathrm{t}, J=7.6 \mathrm{~Hz}, 2 \mathrm{H})$, $2.35(\mathrm{t}, J=7.2 \mathrm{~Hz}, 2 \mathrm{H}), 1.61-1.49(\mathrm{~m}, 13 \mathrm{H}), 0.96(\mathrm{~m}, 6 \mathrm{H}) .{ }^{13} \mathrm{C} \mathrm{NMR}\left(100 \mathrm{MHz}, \mathrm{CDCl}_{3}\right) \delta$ : 150.0, 131.0, 124.9, 119.7, 111.3, 82.8, 28.0, 27.9, 27.7, 23.8, 23.6, 14.1, 14.0. IR (neat, $\left.\mathrm{cm}^{-1}\right): 2960,2932,2871,1741,1500,1457,1418,1369,1334,1255,1173,1143,1107$, 1051, 966, 854. Anal. Calcd for $\mathrm{C}_{15} \mathrm{H}_{25} \mathrm{NO}_{2}$ : C, 71.67; $\mathrm{H}, 10.02$. Found: $\mathrm{C}, 71.39 ; \mathrm{H}, 10.06$. 


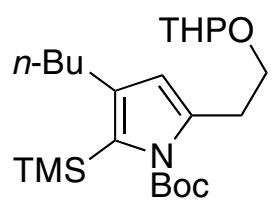

tert-Butyl 3-butyl-5-[2-(tetrahydro-2H-pyran-2-yloxy)ethyl]-2-(trimethylsilyl)-1Hpyrrole-1-carboxylate (Table 1, 2c). Following general procedure A: 1c (0.28 g, 0.50 $\mathrm{mmol})$, for $10 \mathrm{~h}$. Column chromatography: silica gel (2.5\% $\left.\mathrm{NEt}_{3}\right), 10: 1$ hexanes/ethyl acetate. Colorless oil; yield: $0.18 \mathrm{~g}\left(86 \%\right.$ yield). ${ }^{1} \mathrm{H}$ NMR $\left(400 \mathrm{MHz}, \mathrm{CDCl}_{3}\right) \delta: 5.94(\mathrm{~s}, 1 \mathrm{H})$, 4.61 (t, J=2.8 Hz, 1H), 3.97 (q, J=2 Hz, 1H), $3.93(\mathrm{~m}, 1 \mathrm{H}), 3.66$ (q, J=2.2 Hz, 1H), 3.46 (m, $1 \mathrm{H}), 3.08$ (t, J=7.5 Hz, 2H), 2.48 (t, J=7.6 Hz, 2H), 1.93-1.74 (m, 2H), $1.61(\mathrm{~s}, 9 \mathrm{H}), 1.59-$


138.1, 134.4, 129.4, 117.3, 98.8, 83.5, 66.7, 62.3, 34.3, 30.7, 30.6, 30.1, 28.0, 27.6, 22.7, 19.5, 14.1, 2.2. IR (neat, $\mathrm{cm}^{-1}$ ): 2971, 2936, 2873, 1729, 1457, 1369, 1255 1159, 1076, 1034, 977, 906, 869, 813. 

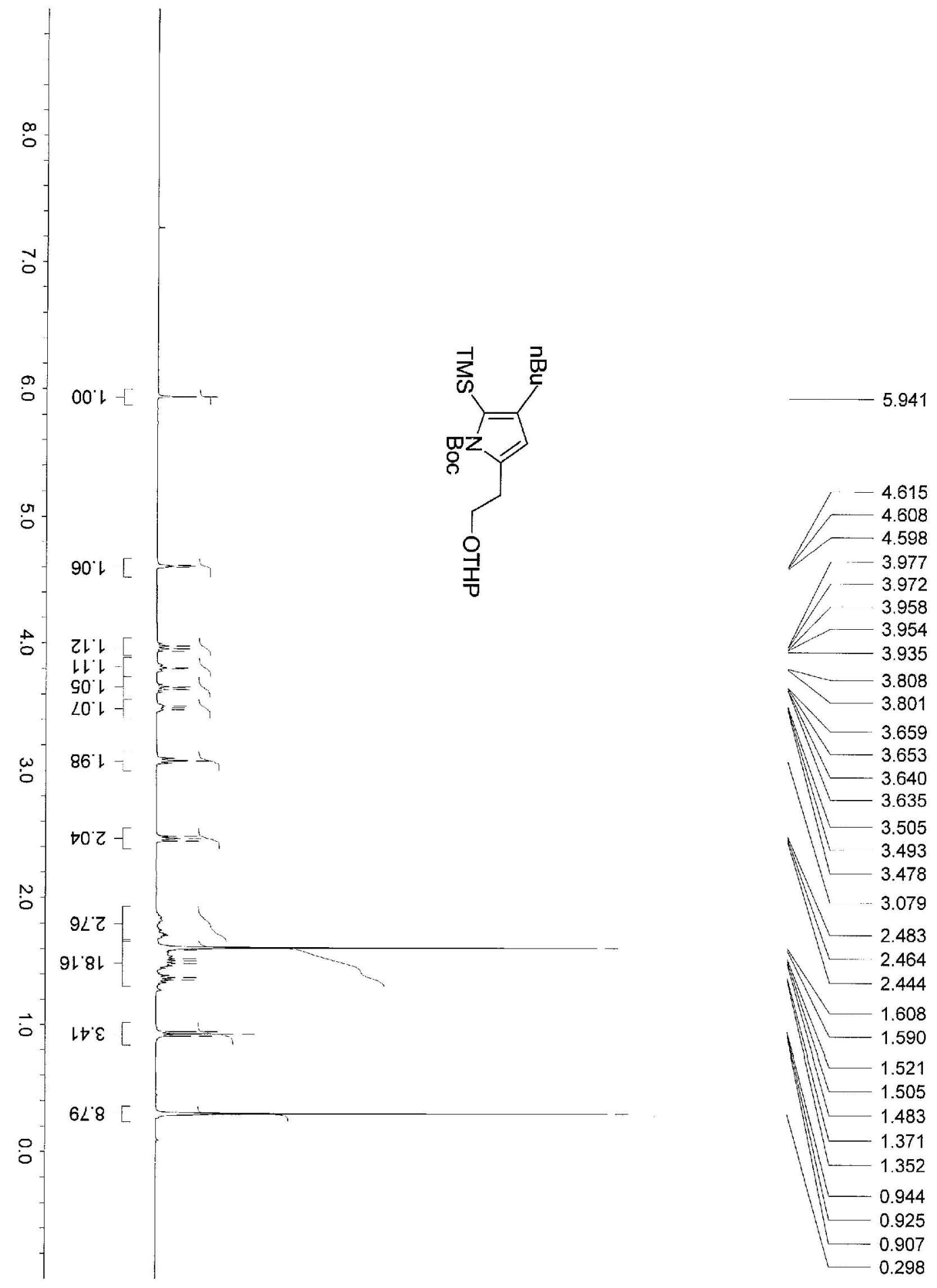


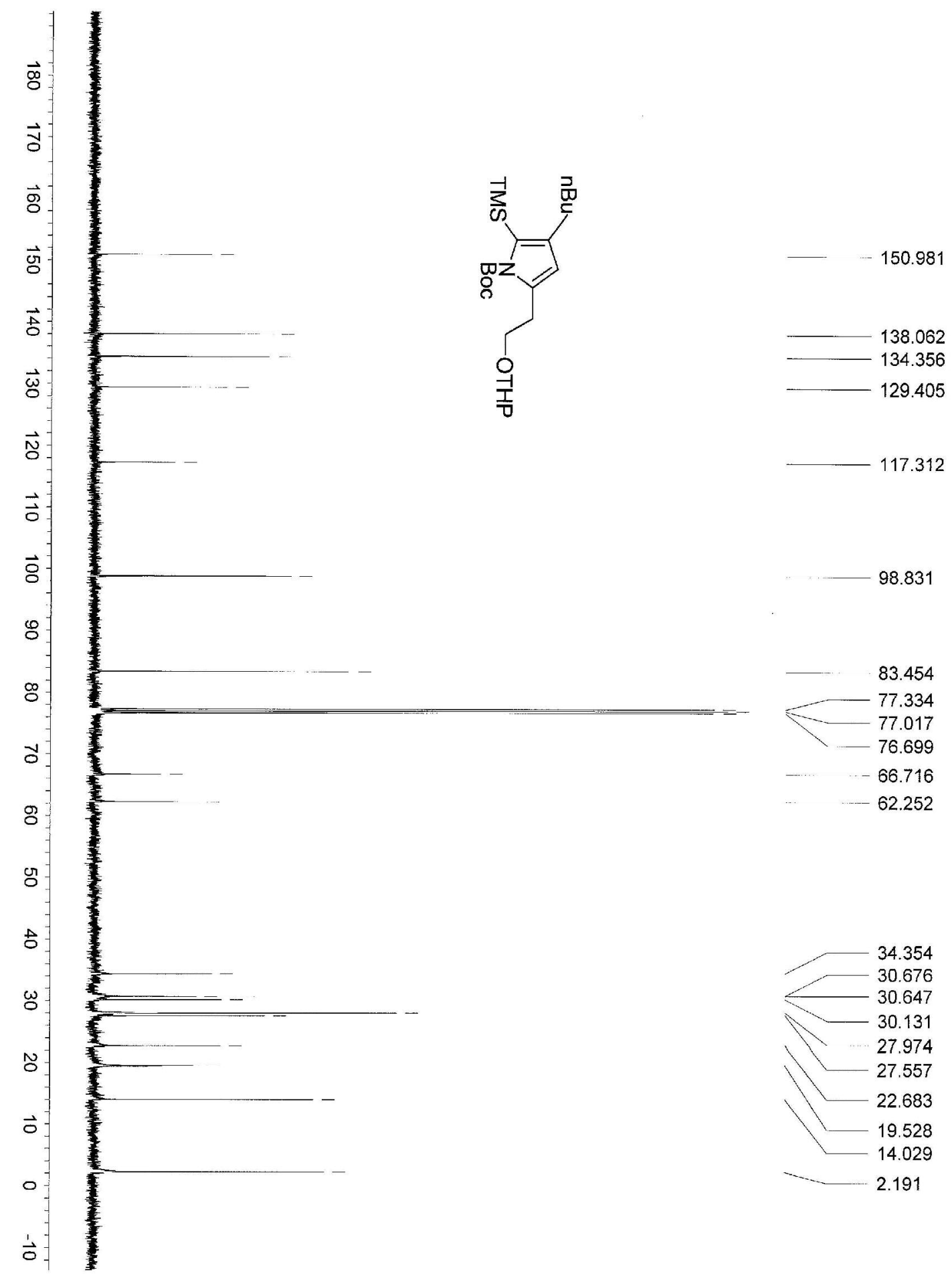






tert-Butyl 3-butyl-5-(4-chlorobutyl)-2-(trimethylsilyl)-1H-pyrrole-1-carboxylate (Table 1, 2d). Following general procedure A: $1 \mathrm{~d}(0.25 \mathrm{~g}, 0.48 \mathrm{mmol})$, for $9 \mathrm{~h}$. Column chromatography: silica gel $\left(2.5 \% \mathrm{NEt}_{3}\right), 10: 1$ hexanes/ethyl acetate . Colorless oil; yield: $0.17 \mathrm{~g}\left(92 \%\right.$ yield). ${ }^{1} \mathrm{H}$ NMR (400 MHz, $\left.\mathrm{CDCl}_{3}\right) \delta: 5.91$ (s, 1H), 3.59 (t, J=6.4 Hz, 2H), 2.79 (t, J=7.6 Hz, 2H), 2.49 (t, J=7.6 Hz, 2H), 1.92-1.86 (m, 2H), 1.84-1.78 (m, 2H), $1.63(\mathrm{~s}, 9 \mathrm{H})$, 1.54-1.39 (m, 4H), $0.96(\mathrm{t}, J=7.2 \mathrm{~Hz}, 3 \mathrm{H}), 0.33(\mathrm{~s}, 9 \mathrm{H}) .{ }^{13} \mathrm{C}$ NMR (100 MHz, $\left.\mathrm{CDCl}_{3}\right) \delta$ : 151.1, 138.1, 137.3, 129.5, 114.3, 83.3, 44.9, 34.4, 32.4, 29.1, 28.0, 27.7, 26.6, 22.8, 14.0, 2.3. IR (neat, $\mathrm{cm}^{-1}$ ): 2955, 2930, 1731, 1580, 1457, 1393, 1369, 1336, 1245, 1134, 1109, 852. Anal. Calcd for $\mathrm{C}_{20} \mathrm{H}_{36} \mathrm{CINO}_{2} \mathrm{Si}$ : C, 62.22; $\mathrm{H}, 9.40$. Found: $\mathrm{C}, 61.98 ; \mathrm{H}, 9.53$.



Di(tert-butyl) 5-butyl-2-[tert-butyl(dimethyl)silyl]-1H-pyrrole-1,3-dicarboxylate (Table 1, 2e). Following general procedure A: 1 e $(0.29 \mathrm{~g}, 0.50 \mathrm{mmol})$, for $8 \mathrm{~h}$. Column chromatography: silica gel $\left(2.5 \% \mathrm{NEt}_{3}\right), 40: 1$ hexanes/ethyl acetate. White solid; yield: $0.22 \mathrm{~g}\left(98 \%\right.$ yield). Mp 62-63 ${ }^{\circ} \mathrm{C} .{ }^{1} \mathrm{H}$ NMR (400 MHz, $\left.\mathrm{CDCl}_{3}\right)$ ס: 6.21 (s, 1H), 2.70 (t, J=7.6 $\mathrm{Hz}, 2 \mathrm{H}), 1.62(\mathrm{~s}, 9 \mathrm{H}), 1.61-1.59(\mathrm{~m}, 2 \mathrm{H}), 1.56(\mathrm{~s}, 9 \mathrm{H}), 1.47-1.39(\mathrm{~m}, 2 \mathrm{H}), 1.10(\mathrm{~s}, 9 \mathrm{H}), 0.97$ (t, J=7.2 Hz, 3H), 0.24 (s, 6H). ${ }^{13} \mathrm{C}$ NMR (100 MHz, $\left.\mathrm{CDCl}_{3}\right)$ ס: 165.3, 150.9, 138.0, 136.8, 131.0, 111.5, 84.4, 79.8, 31.0, 28.8, 28.7, 28.3, 28.0, 22.7, 18.0, 14.0, -1.4. IR (neat, $\mathrm{cm}^{-1}$ ): 2971, 2931, 2858, 1749, 1716, 1471, 1390, 1368, 1317, 1248, 1159, 1131, 1092, 1015. Anal. Calcd for $\mathrm{C}_{24} \mathrm{H}_{43} \mathrm{NO}_{4} \mathrm{Si}: \mathrm{C}, 65.86 ; \mathrm{H}, 9.90$. Found: C, 65.59; $\mathrm{H}, 9.92$.

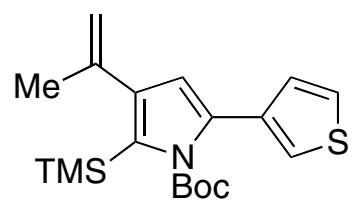

tert-Butyl 3-isopropenyl-5-(3-thienyl)-2-(trimethylsilyl)-1H-pyrrole-1-carboxylate (Table 1, 2f). Following general procedure A: 1f $(0.25 \mathrm{~g}, 0.50 \mathrm{mmol})$, for $10 \mathrm{~h}$. Column chromatography: silica gel $\left(2.5 \% \mathrm{NEt}_{3}\right), 40: 1$ hexanes/ethyl acetate. White solid; yield:

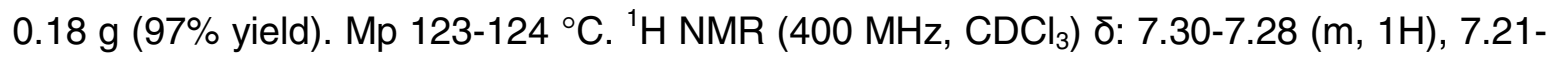
$7.20(\mathrm{~m}, 1 \mathrm{H}), 7.08$ (dd, J=5.2, $1.2 \mathrm{~Hz}, 1 \mathrm{H}), 6.1$ (s, 1H), $5.06(\mathrm{~d}, J=1.2 \mathrm{~Hz}, 1 \mathrm{H}), 4.98$ (d,

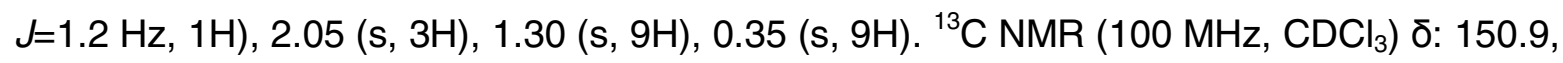
141.0, 140.8, 135.0, 132.2, 130.6, 129.5, 124.0, 122.9, 115.8, 115.0, 83.4, 27.4, 24.8, 1.4. 
IR (neat, cm $\mathrm{cm}^{-1}$ ): 2980, 2943, 1736, 1369, 1326, 1247, 1171, 1149, 1118, 1043, 1011, 895, 848. Anal. Calcd for $\mathrm{C}_{19} \mathrm{H}_{27} \mathrm{NO}_{2} \mathrm{SSi}$ : C, 63.11; $\mathrm{H}, 7.53$. Found: $\mathrm{C}, 63.20 ; \mathrm{H}, 7.51$.

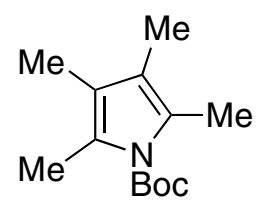

tert-Butyl 2,3,4,5-tetramethyl-1H-pyrrole-1-carboxylate (Table 1, 2g). Following general procedure A: 2,5-diiodo-3,4-dimethyl-hexa-2,4-diene $1 \mathrm{~g}(0.18 \mathrm{~g}, 0.50 \mathrm{mmol})$, for $14 \mathrm{~h}$. Column chromatography: silica gel $\left(2.5 \% \mathrm{NEt}_{3}\right), 50: 1$ hexanes/ethyl acetate. Colorless oil; yield: $0.11 \mathrm{~g}$ (97\% yield). ${ }^{1} \mathrm{H}$ NMR (400 MHz, $\left.\mathrm{CDCl}_{3}\right)$ ס: $2.34(\mathrm{~s}, 6 \mathrm{H}), 1.93(\mathrm{~s}, 6 \mathrm{H}), 1.62$ (s, 9H). ${ }^{13} \mathrm{C}$ NMR (100 MHz, $\left.\mathrm{CDCl}_{3}\right) \delta: 150.6,125.0,118.3,82.5,28.1,13.2,9.3$. IR (neat, $\mathrm{cm}^{-}$ $\left.{ }^{1}\right): 2981,2934,1723,1477,1456,1369,1256,1159,1062,912$, 845. Anal. Calcd for $\mathrm{C}_{13} \mathrm{H}_{21} \mathrm{NO}_{2}$ : C, 69.92; $\mathrm{H}, 9.48$. Found: C, 69.81; $\mathrm{H}, 9.37$.<smiles></smiles>

tert-Butyl 3-butyl-4,5-dipropyl-2-(trimethylsilyl)-1H-pyrrole-1-carboxylate (Table 1, 2h). Following general procedure $A$ : $1 \mathrm{~h}(0.26 \mathrm{~g}, 0.50 \mathrm{mmol})$, for $14 \mathrm{~h}$. Column chromatography: silica gel (2.5\% $\left.\mathrm{NEt}_{3}\right), 50: 1$ hexanes/ethyl acetate. Colorless oil; yield: $0.15 \mathrm{~g}\left(78 \%\right.$ yield). ${ }^{1} \mathrm{H}$ NMR (400 MHz, $\left.\mathrm{CDCl}_{3}\right) \delta: 2.72-2.63(\mathrm{~m}, 2 \mathrm{H}), 2.44(\mathrm{t}, \mathrm{J}=7.6 \mathrm{~Hz}, 2 \mathrm{H})$, 2.32-2.27 (m, 2H), $1.62(\mathrm{~s}, 9 \mathrm{H}), 1.59-1.43(\mathrm{~m}, 8 \mathrm{H}), 1.01-0.97(\mathrm{~m}, 9 \mathrm{H}), 0.36(\mathrm{~s}, 9 \mathrm{H}) .{ }^{13} \mathrm{C}$

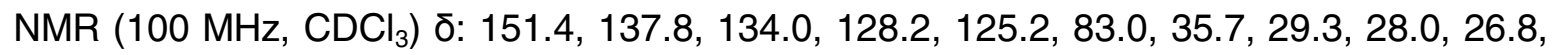
25.4, 24.8, 24.2, 23.1, 14.7, 14.3, 14.0, 2.4. IR (neat, $\mathrm{cm}^{-1}$ ): 2971, 2924, 2867, 1719, 1458, 1366, 1329, 1291, 1107, 1032, 984. Anal. Calcd for $\mathrm{C}_{22} \mathrm{H}_{41} \mathrm{NO}_{2} \mathrm{Si}: \mathrm{C}, 69.60 ; \mathrm{H}, 10.89$. Found: C, 69.79; H, 10.80.<smiles>Cc1c(C(C)(C)C)[nH]c(S(C)(=O)=O)c1C</smiles>

tert-Butyl 3,4-dimethyl-2,5-bis(trimethylsilyl)-1H-pyrrole-1-carboxylate (Table 1, 2i). Following general procedure $A: \mathbf{1 i}^{2}(0.24 \mathrm{~g}, 0.50 \mathrm{mmol})$, for $8 \mathrm{~h}$. Column chromatography: silica gel (2.5\% $\left.\mathrm{NEt}_{3}\right), 40: 1$ hexanes/ethyl acetate. Colorless oil; yield: $0.16 \mathrm{~g}$ (97\% yield). ${ }^{1} \mathrm{H}$ NMR (400 MHz, $\mathrm{CDCl}_{3}$ ) $\delta: 2.11$ (s, 6H), 1.68 (s, 9H), 0.39 (s, 9H). ${ }^{13} \mathrm{C} \mathrm{NMR} \mathrm{(100} \mathrm{MHz,}$ $\mathrm{CDCl}_{3}$ ) $\delta: 152.1,134.5,134.0,85.0,28.5,12.0,2.7$. IR (neat, $\mathrm{cm}^{-1}$ ): 2983, 2949, 1723, 
1479, 1455, 1369, 1331, 1246, 1156, 1076, 984, 874, 844, 772, 678. Anal. Calcd for $\mathrm{C}_{17} \mathrm{H}_{33} \mathrm{NO}_{2} \mathrm{Si}_{2}: \mathrm{C}, 60.12 ; \mathrm{H}, 9.79$. Found: $\mathrm{C}, 59.87 ; \mathrm{H}, 9.84$.

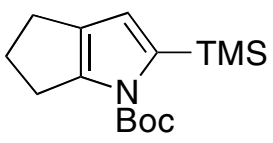

tert-Butyl 2-(trimethylsilyl)-5,6-dihydrocyclopenta[b]pyrrole-1-(4H)-carboxylate (Table 1, 2j). Following general procedure A: $1 \mathbf{j}(0.28 \mathrm{~g}, 0.75 \mathrm{mmol})$, for $6 \mathrm{~h}$. Column chromatography: silica gel $\left(2.5 \% \mathrm{NEt}_{3}\right), 50: 1$ hexanes/ethyl acetate. White solid; yield: $0.20 \mathrm{~g}\left(94 \%\right.$ yield). Mp 51-52 ${ }^{\circ} \mathrm{C} .{ }^{1} \mathrm{H}$ NMR (400 MHz, $\left.\mathrm{CDCl}_{3}\right)$ ס: 6.34 (s, 1H), 2.92 (t, J=7.6 $\mathrm{Hz}, 2 \mathrm{H}), 2.58(\mathrm{t}, \mathrm{J}=7.2 \mathrm{~Hz}, 2 \mathrm{H}), 2.47-2.40(\mathrm{~m}, 2 \mathrm{H}), 1.61(\mathrm{~s}, 9 \mathrm{H}), 0.31(\mathrm{~s}, 9 \mathrm{H}) .{ }^{13} \mathrm{C} \mathrm{NMR}$


$\left(\right.$ neat, $\mathrm{cm}^{-1}$ ): 2971, 2954, 1737, 1371, 1349, 1295, 1245, 1176, 1129, 1112, 843. Anal. Calcd for $\mathrm{C}_{15} \mathrm{H}_{25} \mathrm{NO}_{2} \mathrm{Si}: \mathrm{C}, 64.47 ; \mathrm{H}, 9.02$. Found: $\mathrm{C}, 64.79 ; \mathrm{H}, 8.97$.

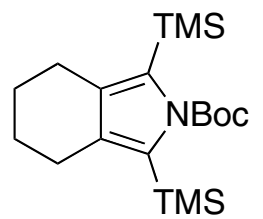

tert-Butyl 1,3-bis(trimethylsilyl)-4,5,6,7-tetrahydro-2 $\mathrm{H}$-isoindole-2-carboxylate (Table 1, 2k). Following general procedure $A: \mathbf{1 k}^{2}(0.25 \mathrm{~g}, 0.50 \mathrm{mmol})$, for $10 \mathrm{~h}$. Column chromatography: silica gel $\left(2.5 \% \mathrm{NEt}_{3}\right), 50: 1$ hexanes/ethyl acetate. White solid; yield: $0.18 \mathrm{~g}\left(99 \%\right.$ yield). Mp 95-96 ${ }^{\circ} \mathrm{C} .{ }^{1} \mathrm{H}$ NMR (400 MHz, $\mathrm{CDCl}_{3}$ ) $\delta: 2.72$ (brs, 4H), 1.74 (brs, $4 \mathrm{H}), 1.68$ (s, 9H), 0.37 (s, 9H). ${ }^{13} \mathrm{C}$ NMR (100 MHz, $\left.\mathrm{CDCl}_{3}\right)$ ठ: 152.1, 135.0, 132.8, 85.2, 28.5, 25.3, 23.6, 2.6. IR (neat, $\mathrm{cm}^{-1}$ ): 2972, 2933, 1724, 1457, 1369, 1332, 1245, 1156, 1058, 970, 843. Anal. Calcd for $\mathrm{C}_{19} \mathrm{H}_{35} \mathrm{NO}_{2} \mathrm{Si}_{2}$ : C, 62.41; $\mathrm{H}, 9.65$. Found: $\mathrm{C}, 62.50 ; \mathrm{H}, 9.61$.

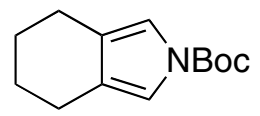

tert-Butyl 4,5,6,7-tetrahydro-2 $\mathrm{H}$-isoindole-2-carboxylate (Table 1, 2l). Following general procedure $A: 1^{6}(0.27 \mathrm{~g}, 0.75 \mathrm{mmol})$, for $7 \mathrm{~h}$. Column chromatography: silica gel (2.5\% $\mathrm{NEt}_{3}$ ), 50:1 hexanes/ethyl acetate. Colorless oil; yield: $0.17 \mathrm{~g}$ (99\% yield). ${ }^{1} \mathrm{H} \mathrm{NMR}$ $\left(400 \mathrm{MHz}, \mathrm{CDCl}_{3}\right) \delta: 6.95$ (s, 2H), 2.58 (brs, 4H), 1.76-1.72 (m, 4H), 1.61 (s, 9H). ${ }^{13} \mathrm{C} \mathrm{NMR}$ $\left(100 \mathrm{MHz}_{\mathrm{CDCl}}\right)$ ) $\mathrm{C:} 149.1,123.6,115.0,82.6,28.0,23.6,22.0$. IR (neat, $\left.\mathrm{cm}^{-1}\right): 2975$, 2932, 2856, 1736, 1526, 1475, 1441, 1406, 1368, 1340, 1322, 1250, 1163, 1057, 978, 857. Anal. Calcd for $\mathrm{C}_{13} \mathrm{H}_{19} \mathrm{NO}_{2}$ : C, 70.56; $\mathrm{H}, 8.65$. Found: $\mathrm{C}, 70.91 ; \mathrm{H}, 8.53$.

\footnotetext{
${ }^{6}$ Xi, Z.; Song, Z.; Liu, G.; Liu, X.; Takahashi, T. J. Org. Chem. 2006, 71, 3154.
} 


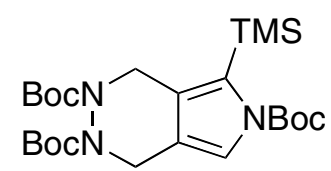

Tri(tert-butyl) 5-(trimethylsilyl)-1 H-pyrrolo[3,4- $d]$ pyridazine-2,3,6(4H)-tricarboxylate (Table 1, 2m). Following general procedure A: $1 \mathrm{~m}(0.35 \mathrm{~g}, 0.50 \mathrm{mmol})$, for $16 \mathrm{~h}$. Column chromatography: silica gel $\left(2.5 \% \mathrm{NEt}_{3}\right), 5: 1$ hexanes/ethyl acetate. Colorless oil; yield: $0.20 \mathrm{~g}\left(78 \%\right.$ yield). ${ }^{1} \mathrm{H}$ NMR (400 MHz, $\mathrm{CDCl}_{3}$ ) (conformers) $\delta: 7.19(\mathrm{~s}, 1 \mathrm{H})$, 5.21-4.92 (m, $2 \mathrm{H}), 4.26-4.10(\mathrm{~m}, 2 \mathrm{H}), 1.58(\mathrm{~s}, 9 \mathrm{H}), 1.49(\mathrm{~s}, 18 \mathrm{H}), 0.31(\mathrm{~s}, 9 \mathrm{H}) .{ }^{13} \mathrm{C} \mathrm{NMR}(100 \mathrm{MHz}$,

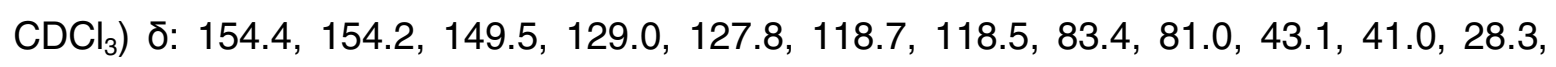
27.9, 1.1. IR (neat, $\mathrm{cm}^{-1}$ ): 2987, 2978, 1743, 1708, 1592, 1406, 1349, 1368, 1324, 1249, 1177, 1053, 1018, 844. Anal. Calcd for $\mathrm{C}_{24} \mathrm{H}_{41} \mathrm{~N}_{3} \mathrm{O}_{6} \mathrm{Si}$ : C, 58.15; H, 8.34. Found: C, 57.79; $\mathrm{H}, 8.51$.

General Procedure B for the synthesis of heteroarylpyrroles and stericallyencumbered compounds through Cu-catalyzed sequential $\mathrm{C}-\mathrm{N}$ bond-forming reactions (Scheme 5). An oven-dried Schlenk tube was charged with Cul (5 mol\%), amine nucleophile (1.2 equiv.), $\mathrm{K}_{2} \mathrm{CO}_{3}$ (3 equiv.), and, if a solid, the 1,4-dihalo-1,3-diene (1 equiv.). The Schlenk tube was capped with a teflon screwcap and then evacuated and backfilled with argon three times. Under a positive pressure of argon, $N, N^{\prime}$ dimethylethylenediamine (20 mol\%) and, if an oil, the 1,4-dihalo-1,3-diene (1 equiv.) were added via syringe, followed by the addition of dioxane or toluene $(0.5 \mathrm{M})$. The tube was sealed and stirred at $110{ }^{\circ} \mathrm{C}$ in a pre-heated oil bath for the indicated period of time. The reaction mixture was cooled to room temperature, diluted with ethyl acetate or diethyl ether, filtered through a plug of celite, concentrated to dryness and purified by column chromatography on silica gel $\left(2.5 \% \mathrm{NEt}_{3}\right)$, eluting with hexanes/ethyl acetate.

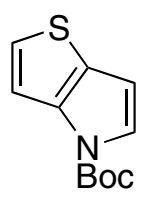


tert-Butyl 4H-thieno[3,2-b]pyrrole-4-carboxylate (Scheme 5, 2n). Following general procedure B: $1 \mathrm{n}^{7}$ (0.20 g, $\left.0.50 \mathrm{mmol}\right)$, for $5 \mathrm{~h}$. Column chromatography: silica gel $(2.5 \%$ $\left.\mathrm{NEt}_{3}\right), 10: 1$ hexanes/ethyl acetate. Colorless oil; yield: $0.11 \mathrm{~g}\left(83 \%\right.$ yield). ${ }^{1} \mathrm{H}$ NMR (400

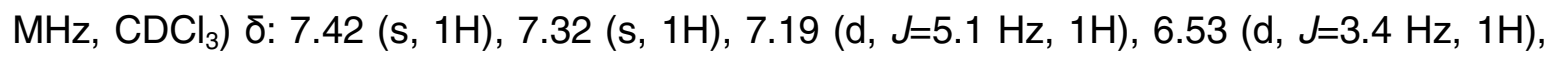
$1.66(\mathrm{~s}, 9 \mathrm{H}) .{ }^{13} \mathrm{C}$ NMR $\left(100 \mathrm{MHz} \mathrm{CDCl}_{3}\right) \delta: 148.9,138.1,127.2,125.2,124.3,115.0$, 105.7, 84.0, 28.5, 28.3, 28.0. IR (neat, $\mathrm{cm}^{-1}$ ): 2978. 2956, 1727, 1421, 1361, 1293, 1260, 1164, 1120, 879, 841, 703. Anal. Calcd for $\mathrm{C}_{13} \mathrm{H}_{13} \mathrm{NO}_{2} \mathrm{~S}_{2}$ : C, 55.89; $\mathrm{H}, 4.69$. Found: C, 55.96; H, 4.78.

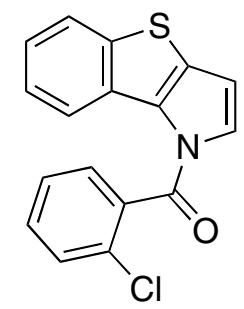

1-(2-chlorobenzoyl)-1H-[1]benzothieno[3,2-b]pyrrole (Scheme 5, 20). Following general procedure B: $10^{7}(0.16 \mathrm{~g}, 0.50 \mathrm{mmol})$, Cul $(5 \mathrm{mg}, 5 \mathrm{~mol} \%)$, 2-chlorobenzamide (93 $\mathrm{mg}, 0.6 \mathrm{mmol})$, for $6 \mathrm{~h}$. Column chromatography: silica gel $\left(2.5 \% \mathrm{NEt}_{3}\right), 20: 1$ hexanes/ethyl acetate. Off-white solid; yield: $0.11 \mathrm{~g}$ (83\% yield). Mp: decomposition (>120 $\left.{ }^{\circ} \mathrm{C}\right) .{ }^{1} \mathrm{H}$ NMR (400 MHz, $\mathrm{CDCl}_{3}$ ) ס: 9.02 (d, J=8.2 Hz, 1H), 7.86 (d, J=8.0 Hz, 1H), 7.57-7.48 (m, 4H), $7.38(\mathrm{~m}, 2 \mathrm{H}), 6.87(\mathrm{~d}, J=3.6 \mathrm{~Hz}, 1 \mathrm{H}), 6.62\left(\mathrm{~d}, J=3.6 \mathrm{~Hz}, 1 \mathrm{H} .{ }^{13} \mathrm{C}\right.$ NMR $\left(100 \mathrm{MHz}, \mathrm{CDCl}_{3}\right) \delta$ : 164.9, 142.6, 134.1, 132.7, 132.0, 131.6, 130.3, 129.6, 129.2, 128.0, 127.4, 127.1, 124.7, 124.3, 124.0, 123.6, 107.6. IR (neat, $\mathrm{cm}^{-1}$ ): 2916, 2851, 1697, 1432, 1342, 1191, 1081, 749. Anal. Calcd for $\mathrm{C}_{17} \mathrm{H}_{10} \mathrm{CINOS}_{2}$ : C, 65.49; H, 3.23. Found: C, 65.49; H, 3.25.

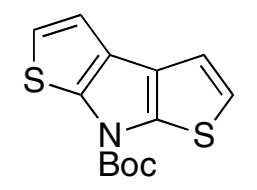

tert-Butyl 7H-dithieno[2,3-b;3,2-d]pyrrole-7-carboxylate (Scheme 5, 2q). Following general procedure B: $1 \mathrm{u}(0.20 \mathrm{~g}, 0.50 \mathrm{mmol})$, Cul $(5 \mathrm{mg}, 5 \mathrm{~mol} \%)$, for $24 \mathrm{~h}$. Column chromatography: silica gel $\left(2.5 \% \mathrm{NEt}_{3}\right), 20: 1$ hexanes/ethyl acetate. White solid; yield: $0.11 \mathrm{~g}\left(78 \%\right.$ yield). $\mathrm{Mp} 72-73{ }^{\circ} \mathrm{C} .{ }^{1} \mathrm{H}$ NMR (400 MHz, $\left.\mathrm{CDCl}_{3}\right) \delta: 7.19$ (d, J=5.2 Hz, 2H), $7.10(\mathrm{~d}, J=5.2 \mathrm{~Hz}, 1 \mathrm{H}), 7.05(\mathrm{~d}, J=5.2 \mathrm{~Hz}, 1 \mathrm{H}), 1.75(\mathrm{~s}, 9 \mathrm{H}) .{ }^{13} \mathrm{C}$ NMR $\left(100 \mathrm{MHz}, \mathrm{CDCl}_{3}\right) \delta$ : 147.8, 137.5, 136.7, 126.2, 126.0, 121.2, 120.6, 117.1, 116.6, 85.4, 28.1. IR (neat, $\mathrm{cm}^{-1}$ ): 2978. 2956, 1727, 1421, 1361, 1293, 1260, 1164, 1120, 879, 841, 703. Anal. Calcd for $\mathrm{C}_{13} \mathrm{H}_{13} \mathrm{NO}_{2} \mathrm{~S}_{2}$ : C, 55.89; $\mathrm{H}, 4$.69. Found: C, 55.96; $\mathrm{H}, 4.78$.

\footnotetext{
${ }^{7}$ According with the literature procedure: Matsumoto, M.; Kuroda, K. Tetrahedron Lett. 1980, 21, 4021
} 


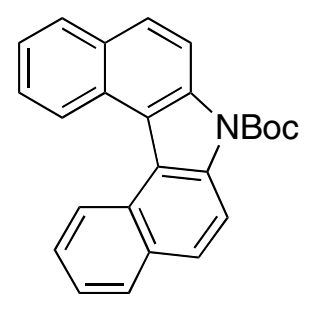

tert-Butyl 7H-dibenzo[c,g]carbazole-7-carboxylate (Scheme 5, 2r). Following general procedure A: 1 r $(0.25 \mathrm{~g}, 0.50 \mathrm{mmol})$, for $24 \mathrm{~h}$. Column chromatography: silica gel $(2.5 \%$ $\mathrm{NEt}_{3}$ ), 40:1 hexanes/ethyl acetate. White solid; yield: $0.14 \mathrm{~g}$ (75\% yield). Mp 114-115 ${ }^{\circ} \mathrm{C}$. ${ }^{1} \mathrm{H}$ NMR (400 MHz, $\mathrm{CDCl}_{3}$ ) ס: 9.06 (d, J=8.3 Hz, 2H), 8.67 (d, J=9.2 Hz, 2H), 8.06 (d, J=8.3 $\mathrm{Hz}, 2 \mathrm{H}), 7.98$ (d, J=9.2 Hz, 2H), 7.65 (t, J=7.2 Hz, 2H), 7.61 (t, J=7.2 Hz), $1.87(\mathrm{~s}, 9 \mathrm{H}) .{ }^{13} \mathrm{C}$ NMR $\left(100 \mathrm{MHz} \mathrm{CDCl}_{3}\right) \delta: 151.6,136.5,131.0,128.6,127.9,127.5,125.9,125.2,124.4$, 120.6, 115.8, 84.8, 28.4. IR (neat, $\mathrm{cm}^{-1}$ ): 2987, 2866, 1732, 1543, 1448, 1391, 1313, 1156, 1114, 804. Anal. Calcd for $\mathrm{C}_{25} \mathrm{H}_{21} \mathrm{NO}_{2}$ : C, 81.72; $\mathrm{H}, 5.76$. Found: $\mathrm{C}, 81.55 ; \mathrm{H}, 5.83$.<smiles>CCCc1ccn(C(=O)O)c1C(=O)O</smiles>

tert-Butyl 2,3-dipropyl-1 H-pyrrole-1-carboxylate (Scheme 3, 2b). TBAF (0.25 mL, 0.25 $\mathrm{mmol}, 1 \mathrm{M}$ THF) was added dropwise to a solution of $\mathbf{2 a}(70 \mathrm{mg}, 0.2 \mathrm{mmol})$ in THF (2 mL) at $0{ }^{\circ} \mathrm{C}$. The mixture was stirred for $8 \mathrm{~h}$ at $70{ }^{\circ} \mathrm{C}$, at which time water $(2 \mathrm{~mL})$ and ethyl acetate $(2 \mathrm{~mL})$ were added. The combined organic layers were dried $\left(\mathrm{MgSO}_{4}\right)$ and evaporated. The product was purified by column chromatography on silica gel $\left(2.5 \% \mathrm{NEt}_{3}\right)$, eluting with hexanes/ethyl acetate $20: 1$ to provide $54 \mathrm{mg}$ of the title compound $(70 \%$ yield) as a colorless oil. ${ }^{1} \mathrm{H}$ NMR $\left(400 \mathrm{MHz}, \mathrm{CDCl}_{3}\right) \delta: 7.17(\mathrm{~d}, J=3.6 \mathrm{~Hz}, 1 \mathrm{H}), 6.02(\mathrm{~d}, J=3.6$ $\mathrm{Hz}, 1 \mathrm{H}), 2.78(\mathrm{t}, J=7.6 \mathrm{~Hz}, 2 \mathrm{H}), 2.35(\mathrm{t}, J=7.2 \mathrm{~Hz}, 2 \mathrm{H}), 1.61-1.49(\mathrm{~m}, 13 \mathrm{H}), 0.96(\mathrm{~m}, 6 \mathrm{H})$.<smiles></smiles>

tert-Butyl 5-lodo-2,3-dipropyl-1H-pyrrole-1-carboxylate (Scheme 3, 2s). An oven-dried Schlenk tube was charged with freshly prepared $2 \mathrm{a}(0.11 \mathrm{~g}, 0.34 \mathrm{mmol})$. The Schlenk tube was capped with a teflon screwcap and then evacuated and backfilled with argon (this sequence was carried out two times). Then $\mathrm{CCl}_{4}(1.5 \mathrm{~mL})$ and $\mathrm{CH}_{2} \mathrm{Cl}_{2}(1.5 \mathrm{~mL})$ were added via syringe, followed by the addition of a solution of $\mathrm{ICl}$ in dichloromethane $1 \mathrm{M}(0.36$ $\mathrm{mL}, 0.36 \mathrm{mmol}$ ) at $-40{ }^{\circ} \mathrm{C}$. The reaction mixture was allowed to stir at $-40{ }^{\circ} \mathrm{C}$ for $1 \mathrm{~h}$. Then, an aqueous saturated solution of $\mathrm{Na}_{2} \mathrm{~S}_{2} \mathrm{O}_{3}(4 \mathrm{~mL})$ and $\mathrm{CH}_{2} \mathrm{Cl}_{2}(4 \mathrm{~mL})$ were added sequentially. The organic phase was separated and washed twice with $\mathrm{H}_{2} \mathrm{O}(5 \mathrm{~mL})$. The 
aqueous layer was separated and back-extracted with $\mathrm{CH}_{2} \mathrm{Cl}_{2}(5 \mathrm{~mL})$, The combined organic layers were dried over $\mathrm{MgSO}_{4}$ and the solvent reduced in vacuo. The product was purified by column chromatography on silica gel $\left(2.5 \% \mathrm{NEt}_{3}\right)$, eluting with hexanes/ethyl acetate $30: 1$ to provide $90 \mathrm{mg}$ of the title compound ( $70 \%$ yield) as a colorless oil. ${ }^{1} \mathrm{H}$ NMR (400 MHz, $\mathrm{CDCl}_{3}$ ) ס: 5.94 (s, 1H), 2.77 (t, J=7.6 Hz, 2H), 2.36 (t, J=7.6 Hz, 2H), 1.62-1.59

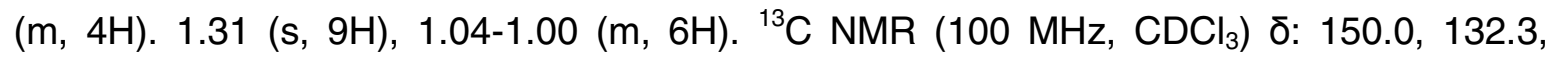
126.3, 123.1, 114.9, 82.2, 28.2, 27.9, 27.6, 24.0, 23.8, 14.2, 14.1. IR (neat, $\mathrm{cm}^{-1}$ ): 2963 , 2913, 2856, 1729, 1456, 1401, 1356, 1299, 1256, 1176, 1168, 1143, 1093, 893, 839. 




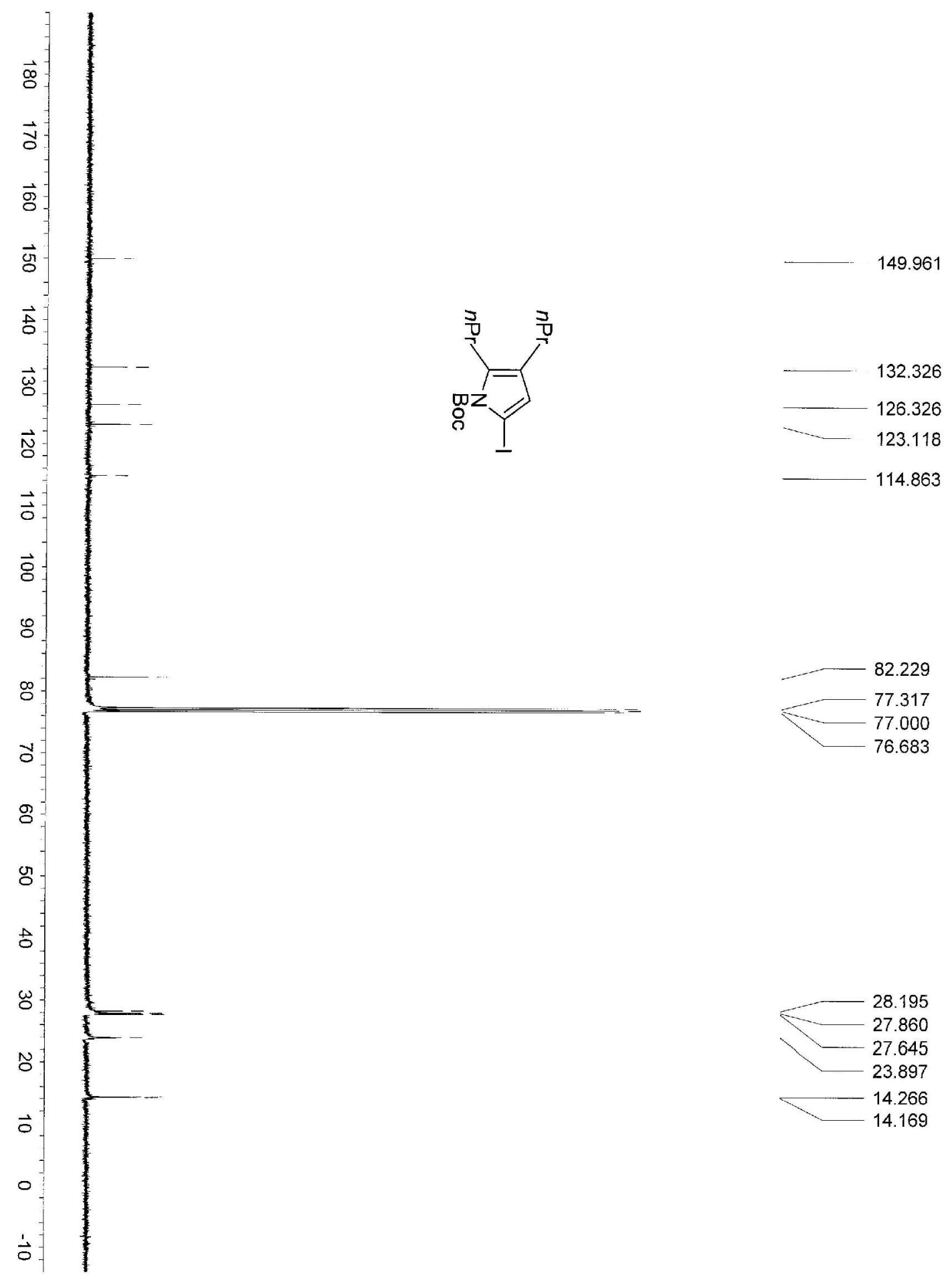

